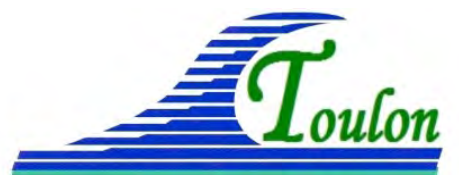

XIV èmes Journées Nationales Génie Côtier - Génie Civil

Toulon, 29 juin au $1^{\text {er }}$ juillet 2016

DOI:10.5150/jngcgc.2016.066 (C) Editions Paralia CFL

disponible en ligne - http://www.paralia.fr - available online

\title{
Sédiments marins traités en grand volume avec de la cendre volante papetière
}

\author{
Xavier PELLERIN LE BAS ${ }^{1}$, Beatriz BOULLOSA-ALLARIZ ${ }^{1}$, \\ Daniel LEVACHER ${ }^{1}$
}

\author{
1. Université de Caen Normandie, Faculté des Sciences, UMR 6143 CNRS - M2C, \\ Esplanade de la Paix, 14032 Caen, France. \\ xavier.pellerin-lebas@unicaen.fr ; beatriz.boullosa-allariz@unicaen.fr ; \\ daniel.levacher@unicaen.fr
}

\section{Résumé :}

Les dépôts de sédiments marins pour une gestion à terre sont importants en MancheMer du Nord, ils avoisinent les 240000 tonnes/année en moyenne sur 2009-2011 excepté les travaux réalisés en 2009 sur Port 2000 au Havre. De plus, les préoccupations environnementales tendent à réduire la quantité de sédiments pouvant être immergée, il est donc nécessaire de trouver de nouvelles filières permettant la valorisation des matériaux non immergeables, tout en garantissant qu'aucune contamination ne soit transmise au milieu récepteur.

Considérant de tels volumes pour une valorisation matière, les mélanges doivent intégrer des pourcentages élevés de sédiments fins ce qui rend leur stabilisation difficile à moins d'augmenter le taux de liants. Il est possible de substituer pour partie ces liants par des coproduits industriels ayant des propriétés liantes comme la cendre volante papetière. On parlera de covalorisation de sédiments et de coproduits, technique qui est de loin plus économique et acceptable du point de vue environnemental.

Des formulations ont été étudiées tout en limitant le dosage en liants classiques avec un sédiment marin. Pour tous les mélanges, une grande majorité de sédiments ( $\geq 80 \%)$ a été incorporée.

Après avoir rappelé les différentes caractéristiques des constituants, les formulations réalisées sont présentées. La détermination des propriétés liantes des formulations (temps de prise, consistance) et de durcissement (résistance, retrait) est faite et les résultats analysés. Les premières conclusions montrent que les sédiments stabilisés ont une résistance à la compression relativement faible pour un matériau routier mais suffisante pour un matériau de remblai.

Mots-clés : Sédiments de dragage, Cendre volante papetière, Stabilisation, Matériau.

\section{Introduction}

Le contexte de cette étude est la valorisation des sédiments marins. Ces derniers représentent un volume important de matériaux à gérer chaque année en France. La grande majorité est rejetée en mer, si leurs niveaux de contamination sont au-dessus des 


\section{Thème 6 - Gestion durable des zones littorales et estuariennes}

textes réglementaires, ils sont stockés à terre. Une des voies de valorisation envisageables est celle des matériaux de construction tels que les briques ou pavés. A ce propos, une étude a été réalisée dans l'optique d'obtenir un mélange qui puisse convenir à la fabrication de produits de construction manufacturés à partir de sédiments. L'objectif a été d'analyser les performances mécaniques des formulations réalisées à partir de coproduits que sont les sédiments fins et des cendres volantes papetières. Les coproduits sont définis comme étant des produits annexes dont la fabrication lors d'un procédé industriel n'est pas intentionnel, mais qui peuvent être valorisés économiquement. Les cendres volantes papetières sont les résidus de chaudière biomasse brûlant des boues de papiers désencrés et du bois de récupération.

Les techniques de stabilisation et de solidification ont été utilisées afin de capturer les contaminants au sein de ces produits manufacturés. Leur efficacité est satisfaisante puisque ces procédés permettent de diminuer de 95\% les concentrations des contaminants dans les lixiviats après traitement des sols (COLOMBANO et al., 2010). De plus ces techniques peuvent améliorer les caractéristiques géotechniques et mécaniques des sédiments par ajout de liants comme le ciment, la chaux ou les coproduits pouvant avoir des propriétés pouzzolaniques tels que les cendres volantes papetières.

\section{Matériaux et méthodes}

Dans cette partie, sont présentés les différents matériaux et produits utilisés. Leurs caractéristiques principales ont été déterminées soit au laboratoire M2C de Caen, soit à l'IFSTTAR de Nantes, (PELLERIN LE BAS, 2015 ; PELLERIN LE BAS et al., 2016).

\subsection{Les sédiments de Dunkerque}

Les sédiments du Port de Dunkerque utilisés dans le cadre de cette étude ont été prélevés en 2015. Plusieurs échantillons ont été réalisés, certains ont été extraits de deux casiers des chambres de décantation du port. Selon le point de prélèvement, les teneurs en eau moyennes varient de $75,5 \%$ à $125 \%$.

La granulométrie des sédiments de Dunkerque s'étale de 2 à $400 \mu \mathrm{m}$. Le $\mathrm{D}_{50}$ est de 18 $\mu \mathrm{m}$, il s'agit donc d'un limon sableux étalé et mal gradué avec une proportion d'argile d'environ 7\%. D’après le Guide des Terrassements Routiers (GTR, 2000), ce matériau est un sol fin et fait donc partie de la classe A. Le graphique de la figure 1 présente la moyenne des volumes différentiels et cumulés en fonction des tailles de particules. 


\section{XIV vèmes Journées Nationales Génie Côtier - Génie Civil \\ Toulon, 29 juin au $1^{\text {er }}$ juillet 2016}

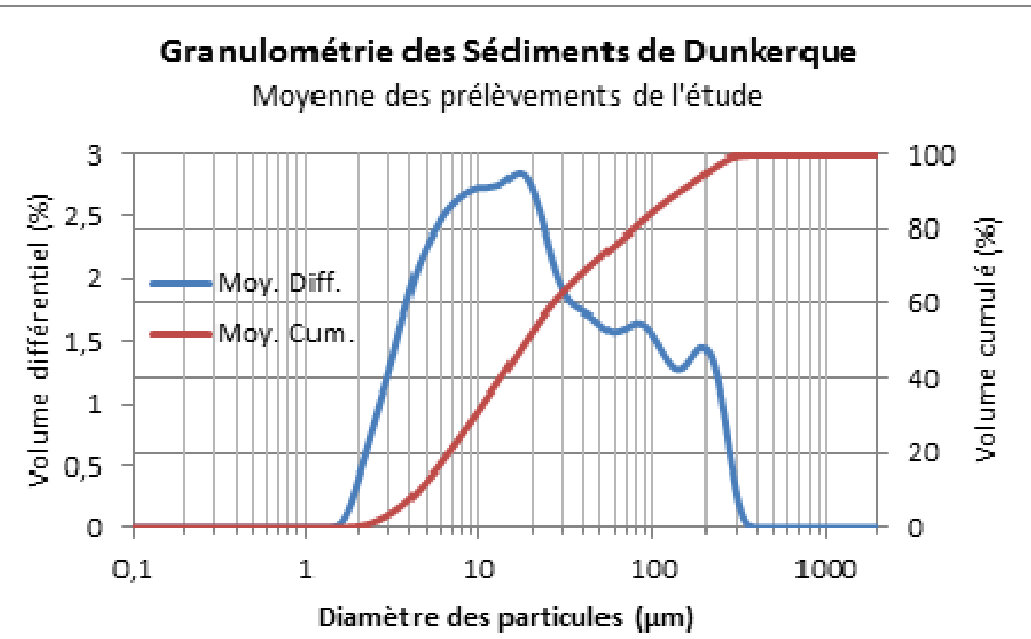

Figure 1. Granulométrie moyenne des sédiments de Dunkerque.

La moyenne globale de teneur en matière organique des prélèvements est de 12,83\%. Ce sont donc des sédiments riches en matière organique. La matière organique peut affecter le processus de cimentation dans les sédiments. En effet, il semble que certains composés organiques retardent ou même empêchent le processus d'hydratation du ciment, alors que d'autres n'affectent aucunement la réaction selon TREMBLAY et al., (2002). La teneur en carbonate de calcium est un indicateur de la résistance mécanique des sédiments ainsi que sa sensibilité à l'eau. La composition en carbonate de calcium des sédiments de Dunkerque est en moyenne de 8,36\%. Une composition chimique plus complète serait nécessaire pour envisager une valorisation matière détaillée.

La dernière caractéristique des sédiments de Dunkerque est leur valeur au bleu de méthylène. Cette dernière va permettre de traduire la sensibilité à l'eau des 7\% d'argile contenus dans les sédiments. La valeur moyenne à l'essai est de 4,1 ce qui correspond à un sol limoneux de plasticité moyenne (NF P 94-068). L'argile contenue dans les sédiments est une argile active, donc sensible à l'eau. Il faudra s'attendre à des phénomènes de retrait et de gonflement en fonction de la teneur en eau des sédiments.

\subsection{Les cendres volantes papetières}

L'addition de ces cendres volantes à des déchets d'origine minérale a pour but d'augmenter la résistance à la compression. Comme le démontrent les travaux présentés dans la thèse de TRAN (2009), un mélange de sédiments et ciment voit sa résistance à la compression à 90 jours tripler par l'ajout de $10 \%$ de cendres volantes. Ce résultat est aussi vrai pour les sédiments seuls, d'après les mêmes travaux. C'est la présence de chaux vive dans les cendres volantes qui permet d'en activer les propriétés pouzzolaniques. Les cendres volantes papetières -CVP- utilisées pour cette étude proviennent de l'entreprise UPM-Chapelle Darblay. Elles se présentent sous la forme d'une poudre brunâtre inodore et compacte. Leur granulométrie s'étale de 2 à $1000 \mu \mathrm{m}$ 


\section{Thème 6 - Gestion durable des zones littorales et estuariennes}

et possède un $\mathrm{D}_{50}$ de $290 \mu \mathrm{m}$. La masse volumique des CVP est d'environ 1,6 t/m $\mathrm{m}^{3}$. La surface spécifique des CVP est comprise entre 500 et $1250 \mathrm{~m}^{2} / \mathrm{kg}$, (ZMAMOU, 2015). Malgré une importante proportion de chaux vive, supérieure à $30 \%$, il apparaît que seul un pourcentage de 0 à $3 \%$ de cette chaux est libre, c'est-à-dire capable de réagir naturellement en présence d'eau.

Etant des résidus de combustion, les CVP sont considérées comme matériau anhydre. Pour la même raison, il n'y a pas de matière organique, et le taux de carbonate de calcium est estimé inférieur à 3\%.

\subsection{Les liants}

Le principal liant utilisé a été un ciment Portland CEMII/A-LL 42,5 (NF EN 197-1). C'est un ciment courant dans les constructions civiles et dans les conceptions d’ouvrages en béton armé. Il offre une classe de résistance de 42,5 MPa à 28 jours.

La chaux vive utilisée est une chaux commerciale en poudre blanche, stockée dans un seau hermétique.

Un liant commercial destiné à la stabilisation des sols, élaboré à base de matières pouzzolaniques, appelé ACTi $200^{\circledR}$ a été utilisé. D’une masse volumique de $2,7 \mathrm{~g} / \mathrm{cm}^{3}$ et d'une densité apparente de 0,65, sa surface spécifique (ou Blaine) est de l'ordre de $12000 \mathrm{~cm}^{2} / \mathrm{g}$, ce qui est largement supérieur au ciment (entre 2800 et $5000 \mathrm{~cm}^{2} / \mathrm{g}$ ).

\subsection{Les formulations}

Les formulations établies ont toutes été réalisées avec une grande proportion de sédiments du port de Dunkerque DK ( $\geq 80 \%)$. En premier lieu, une formulation témoin TEM composé uniquement de sédiments a été faite afin d'avoir des repères quant au retrait lors de la cure de 28 jours, ainsi que pour la résistance mécanique à la compression simple.

Les proportions des mélanges s'entendent en pourcentages massiques, les teneurs en eau ou rapport eau/masse sèche $(E /(D K+\ldots))$ correspondent à des pâtes de consistances normalisées, évaluées à l'appareil de Vicat (NF P 94-050).

Par la suite deux mélanges ont été fabriqués à partir de sédiments, CVP et ACTi $200^{\circledR}$ :
a) DKCVP : Sédiments 91\% + CVP 9\%
$\mathrm{E} /(\mathrm{DK}+\mathrm{CVP})=1,08$
b) DKAc : $\quad$ Sédiments $91 \%+$ ACTi $200^{\circledR} 9 \%$
$\mathrm{E} /(\mathrm{DK}+\mathrm{ACTi} 200 \otimes)=0,95$

Ces deux mélanges possédant des temps de prise extrêmement longs, ils ont chacun été décliné en 3 variantes, en leur ajoutant différentes proportions de ciment, voir tableau 1. A titre d'exemple dans le cas de la formulation DKCVP-2Ci, pour $91 \mathrm{~g}$ de sédiments et $9 \mathrm{~g}$ de cendres volantes papetières i.e. $100 \mathrm{~g}$, on ajoute $2 \mathrm{~g}$ de ciment, ce qui donne $102 \mathrm{~g}$ pour le mélange. Dans ce cas, 102 g devient la référence des dosages pour la formule. Ce sera le cas pour les formules du tableau 1. Les temps de prise étant toujours trop élevés, 5 autres mélanges ont été réalisés afin de pouvoir étudier l’influence des différents éléments. Les compositions de ces derniers sont présentées dans le tableau 2. 
Tableau 1. Composition des variantes aux mélanges DKCVP et DKAc.

\begin{tabular}{lllll}
\hline Formulations & $\begin{array}{l}\text { Sédiments de } \\
\text { Dunkerque }\end{array}$ & CVP & ACTi 200® & Ciment \\
\hline$D K C V P-1 C i$ & $91 \%$ & $9 \%$ & - & $+1 \%$ \\
$D K C V P-2 C i$ & $91 \%$ & $9 \%$ & - & $+2 \%$ \\
$D K C V P-5 C i$ & $91 \%$ & $9 \%$ & - & $+5 \%$ \\
$D K A c-1 C i$ & $91 \%$ & - & $9 \%$ & $+1 \%$ \\
$D K A c-2 C i$ & $91 \%$ & - & $9 \%$ & $+2 \%$ \\
$D K A c-5 C i$ & $91 \%$ & - & $9 \%$ & $+5 \%$ \\
\hline
\end{tabular}

Tableau 2. Composition des 5 formulations.

\begin{tabular}{lllllll}
\hline Formulations & $\begin{array}{l}\text { Eau/ } \\
\text { Masse sèche }\end{array}$ & Sédiments & CVP & Ciment & $\begin{array}{l}\text { Sable } \\
\mathbf{0 / 4 m m}\end{array}$ & Chaux \\
\hline $\mathrm{DKCi}$ & 1,15 & $91 \%$ & - & $9 \%$ & - & - \\
$\mathrm{DK} 2 \mathrm{CVP}$ & 1,06 & $80 \%$ & $20 \%$ & - & - & - \\
$D K C V P S a$ & 1,01 & $80 \%$ & $10 \%$ & - & $10 \%$ & - \\
$D K C i C x$ & 1,05 & $94 \%$ & - & $3 \%$ & - & $3 \%$ \\
$D K 2 C i C x$ & 1,05 & $91 \%$ & - & $6 \%$ & - & $3 \%$ \\
\hline
\end{tabular}

\section{Résultats}

\subsection{Les temps de prise}

Les essais de prise de toutes les formulations ont révélé des temps trop importants, le début de prise arrivant au plus tôt 16 heures après moulage. Il est à noter cependant, que les différents éléments ajoutés aux sédiments ont eu un effet positif sur le temps de prise, car le temps de séchage des sédiments seuls ne débute qu’à partir de 5 jours et ne prend fin qu'après plus de 16 jours de cure à $20^{\circ} \mathrm{C}$.

\subsection{Les retraits dimensionnels}

Les calculs de retrait ont été effectués en s’inspirant de la norme NF P15-433 (1994) à l'aide d'éprouvettes cylindriques utilisées pour les essais de compression simple. Elles mesurent initialement $68 \mathrm{~mm} \pm 0,5 \mathrm{~mm}$ de hauteur et $34 \mathrm{~mm} \pm 0,1 \mathrm{~mm}$ de diamètre. Les dimensions ont été prises immédiatement après démoulage des éprouvettes et juste avant l'essai de compression simple.

Soit $\mathrm{H}$ et $\mathrm{D}$ : hauteur et diamètre initiaux ; h et $\mathrm{d}$ : hauteur et diamètre après 28 jours de cure. On peut exprimer :

a) le retrait relatif sur la hauteur : Rh $(\%)=(\mathrm{H}-\mathrm{h}) \times(100 / \mathrm{H})$

b) le retrait relatif sur le diamètre : $R d(\%)=(D-d) \times(100 / D)$

c) le retrait relatif : $\mathrm{R}(\%)=(\mathrm{Rh}+\mathrm{Rd}) / 2$ 
Les figures 2 et 3 présentent les résultats des retraits relatifs moyens pour toutes les formulations étudiées.

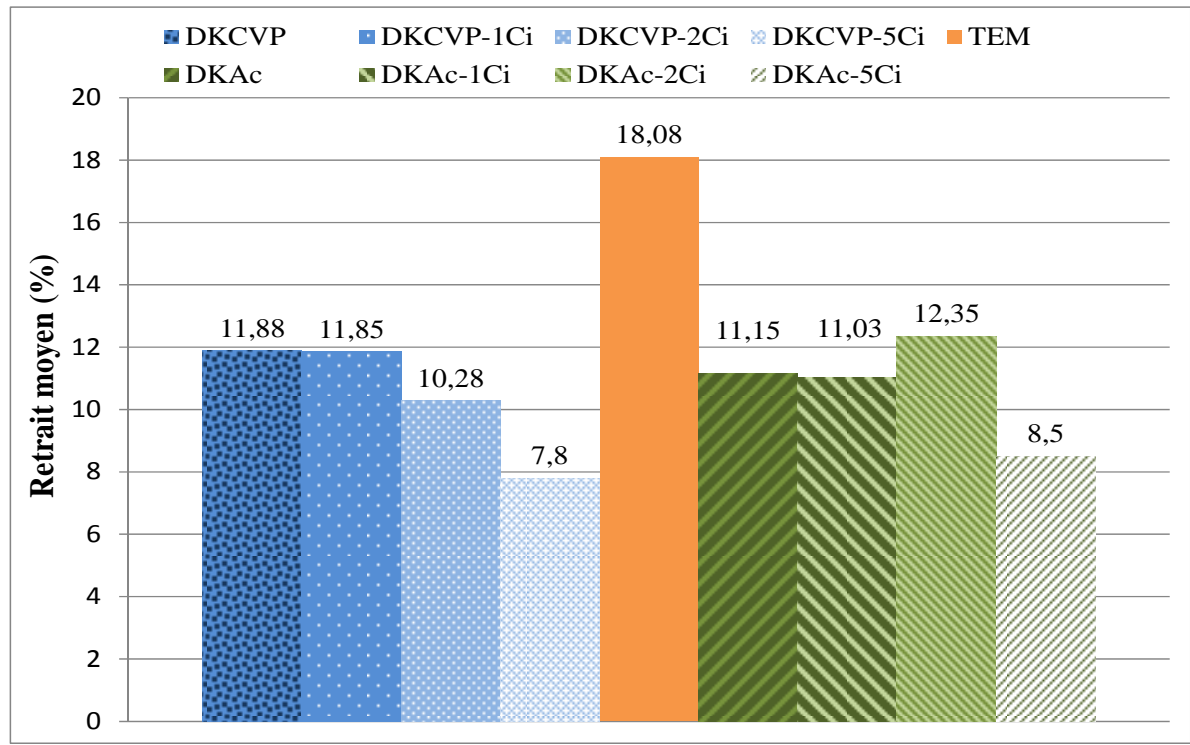

Figure 2. Retraits moyens des formulations DKCVP, DKAc et leurs dérivés.

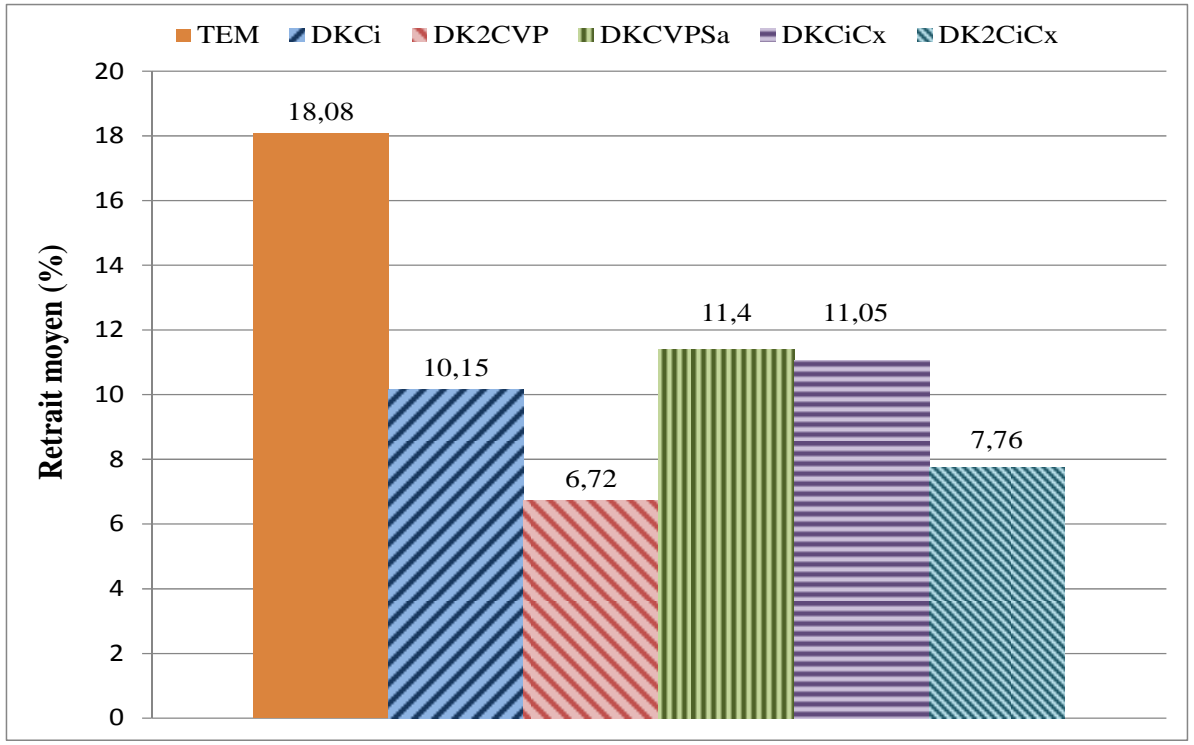

Figure 3. Retraits moyens des 5 formulations et influence des produits ajoutés aux sédiments de Dunkerque.

Les retraits les plus faibles de la figure 2 sont ceux des mélanges contenant $5 \%$ de ciment, avec un retrait d'environ 8\%. La figure 3 montre l'influence des CVP avec le retrait le plus faible (6,7\%) de la formulation DK2CVP (20\% de CVP). La formulation DK2CiCx (6\% ciment et 3\% chaux) présente un retrait d’environ 7,8\%. 


\section{XIV ${ }^{\text {èmes }}$ Journées Nationales Génie Côtier - Génie Civil \\ Toulon, 29 juin au $1^{\text {er }}$ juillet 2016}

\subsection{Résistances à la compression simple}

Les résultats de résistance à la compression simple présentés sont les résistances maximales issues des courbes de contrainte en fonction de la déformation des éprouvettes. Ces courbes ont été obtenues à partir de 3 éprouvettes chacune. La figure 4 ne tient pas compte de toutes les formulations dérivant des mélanges DKCVP et DKAc mais seulement celles possédant les meilleurs résultats.

La grande majorité des formulations testées donne des résultats de résistance maximale à la compression relativement proche des résultats des sédiments seuls, c'est-à-dire entre 0,35 et 0,50 MPa. Le mélange DKAc-5Ci semble se démarquer avec une résistance à la compression se rapprochant de 1,0 $\mathrm{MPa}(0,95 \mathrm{MPa})$. Il est a noté aussi que le fait d'avoir doublé la proportion de CVP dans DK2CVP par rapport à DKCVP semble avoir réduit de moitié les performances mécaniques de DK2CVP.

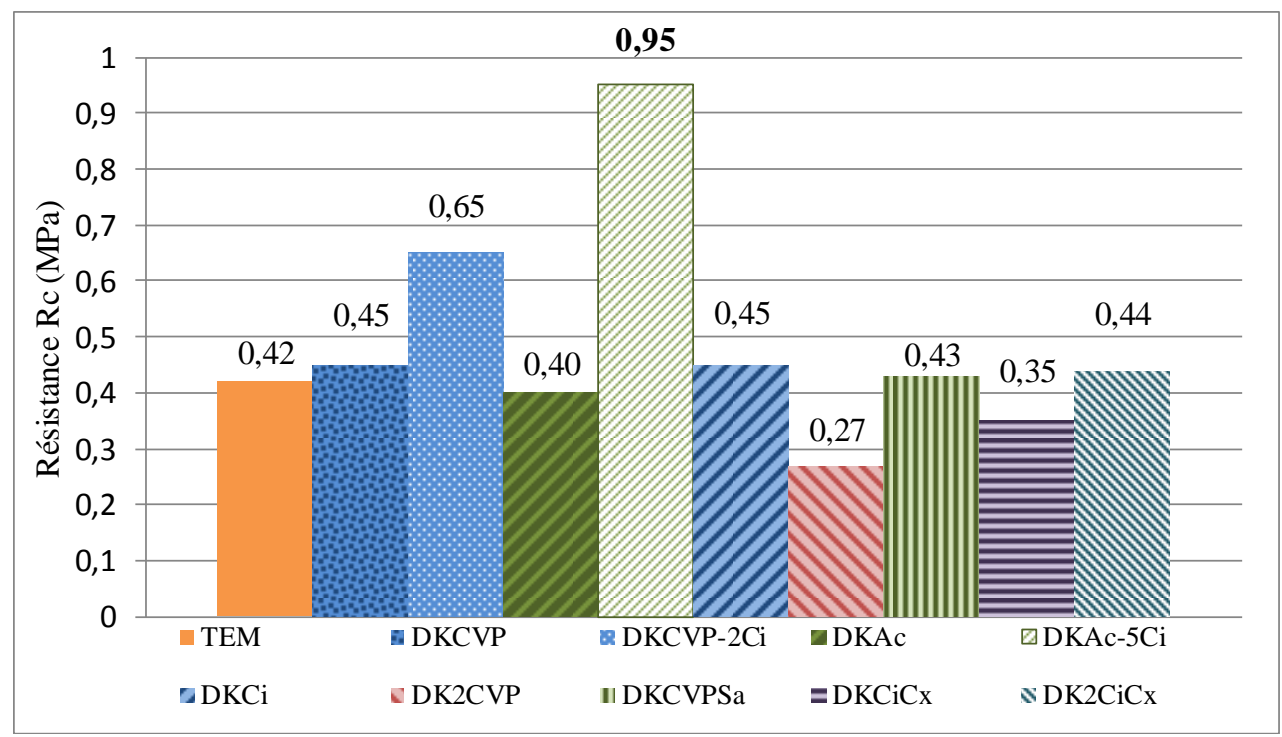

Figure 4. Résistance maximale à la compression simple.

\section{Conclusions}

Cette étude tend à montrer la difficulté de trouver de nouvelles voies de valorisation pour les grandes quantités de sédiments à gérer à terre. L'objectif était d'analyser les performances mécaniques de mélanges à forte proportion de sédiments $(\geq 80 \%)$ dans le but de réaliser des matériaux de construction comme des briques ou pavés. La majorité des formulations testées ont donné des résultats insuffisants aussi bien en termes de temps de prise, de retrait ou de résistance à la compression. En revanche, si l'on réoriente la voie de valorisation de ces sédiments, certains mélanges de cette étude pourraient permettre à de grands volumes de sédiments d'être valorisés en tant que matériau de remblai. C'est par exemple le cas pour la formulation DKAc-5Ci qui présente la meilleure résistance à la compression avec 0,95 $\mathrm{MPa}$, et un retrait parmi les 
plus faibles de $8,5 \%$. L'avantage de la valorisation en tant que remblai est que la teneur en eau pourrait être abaissée car il ne serait plus nécessaire de mouler le matériau sur un laps de temps assez long sans pour autant être un handicap à l'utilisation du matériau, ceci diminuerait le temps de prise. Il pourrait être intéressant d'effectuer un prétraitement à la chaux sur les sédiments de Dunkerque, ce qui aurait le double objectif de lutter contre la forte proportion de matière organique et sur la teneur en eau initiale des sédiments. Ces effets resteraient à démontrer.

\section{Références bibliographiques}

COLOMBANO S., SAADA A., GUERIN V., BATAILLARD P., BELLENFANT G., BERANGER S., HUBE D., BLANC C., ZORNIG C., GIRARDEAU I. (2010). Quelles techniques pour quels traitements - Analyses coûts-bénéfices. Rapport final, BRGM/RP-58609-FR, 399 p.

GTR - Guide des Terrassements Routiers. (2000). Guide technique pour la réalisation des remblais et des couches de forme. Fascicule I \& II, Editions du Setra-LCPC, 200 p. NF EN 197-1. (2012). Ciment - Partie 1 : composition, spécifications et critères de conformité des ciments courants.

NF P15-433. (1994). Méthodes d'essais des ciments - Détermination du retrait et du gonflement.

NF P 94-050. (1995). Sols : Reconnaissance et essais - Détermination de la teneur en eau pondérale des matériaux - Méthode par étuvage.

NF P 94-068. (1998). Sols: Reconnaissance et essais - Mesure de la capacité d'absorption de bleu de méthylène d'un sol ou d'un matériau rocheux - Détermination de la valeur de bleu de méthylène d'un sol ou d'un matériau par essai à la tâche.

PELLERIN LE BAS X. (2015) Valorisation des sédiments du port de Dunkerque en matériaux de construction. Rapport de Master 2, Université du Havre Normandie, 60 p.

PELLERIN LE BAS X., LEVACHER D., BOULLOSA-ALLARIZ B. (2016). Marine sediments paper treated with wastepaper fly ash in a mortar approach. GeoEnvironmental Engineering, GEE 2016, Nantes, France, June 1-2, 2016, in progress.

TRAN N.T. (2009). Valorisation de sédiments marins et fluviaux en technique routière. Thèse, Ecole des Mines de Douai, 187 p.

TREMBLAY H., DUCHESNE J., LOCAT J., LEROUEIL S. (2002). Influence of the nature of organic compounds on fine soil stabilization with cement. Canadian Geotechnical Journal, Vol. 39(3), pp. 535-546. http://dx.doi.org/10.1139/t02-002

ZMAMOU H. (2015). Ecoconception de nouveaux agromatériaux à partir de cendre de chaudière biomasse. Relation structures-propriétés. Thèse, Université de Rouen, $288 \mathrm{p}$. 